Research Article

\title{
Hermite-Hadamard-Type Inequalities for Product of Functions by Using Convex Functions
}

\author{
Tariq Nawaz, ${ }^{1}$ M. Asif Memon $\mathbb{D}^{2},{ }^{2}$ and Kavikumar Jacob $\mathbb{D}^{3}$ \\ ${ }^{1}$ Department of Mathematics, Govt College (B), Dhoke Syedan, Rawalpindi, Pakistan \\ ${ }^{2}$ Department of Mathematics and Social Sciences, Sukkur IBA University, Sukkur 65200, Sindh, Pakistan \\ ${ }^{3}$ Department of Mathematics and Statistics, Faculty of Applied Sciences and Technology, Universiti Tun Hussein Onn, \\ Malaysia Batu Pahat 86400, Johar, Malaysia \\ Correspondence should be addressed to Kavikumar Jacob; kavi@uthm.edu.my
}

Received 29 October 2020; Revised 4 January 2021; Accepted 13 January 2021; Published 27 January 2021

Academic Editor: Imtiaz Ahmad

Copyright (C) 2021 Tariq Nawaz et al. This is an open access article distributed under the Creative Commons Attribution License, which permits unrestricted use, distribution, and reproduction in any medium, provided the original work is properly cited.

\begin{abstract}
One of the many techniques to obtain a new convex function from the given functions is to calculate the product of these functions by imposing certain conditions on the functions. In general, the product of two or finite number of convex function needs not to be convex and, therefore, leads us to the study of product of these functions. In this paper, we reframe the idea of product of functions in the setting of generalized convex function to establish Hermite-Hadamard-type inequalities for these functions. We have analyzed different cases of double and triple integrals to derive some new results. The presented results can be viewed as the refinement and improvement of previously known results.
\end{abstract}

\section{Introduction}

Theory of convex functions has an essential role in different areas of mathematics, especially in optimization and modern analysis. Convex functions have many unique properties, for example, if a function is strictly convex, then it has a unique minimum on an open set. Even when the dimension of space is not finite, convex functions posses the similar properties and as a consequence, they are the examples of functionals in variation methods. A convex function via a random variable is bounded above by the expected value in the theory of probability. In fact, this is Jensen's inequality, which can be used to reobtain many inequalities like the arithmeticgeometric mean inequality and Hölder's inequality. One of the very fundamental results regarding convexity is the wellreputed Hermite-Hadamard inequality.

$$
p\left(\frac{\xi_{1}+\xi_{2}}{2}\right) \leq \frac{1}{\xi_{2}-\xi_{1}} \int_{\xi_{1}}^{\xi_{2}} p(x) \mathrm{d} x \leq \frac{p\left(\xi_{1}\right)+p\left(\xi_{2}\right)}{2} .
$$

Convexity has been generalized in many aspects, and the classical Hermite-Hadamard inequality is viewed by these generalizations. In [1], Toader extended the idea of convexity by giving the definition of an $m$-convex function and constructed few results including Hermite-Hadamard-type inequalities. Further development on $m$-convex and $(\alpha, m)$-convex functions can be noticed in [1-9] and references therein. Further results on convex, $m$-convexn and $(\alpha, m, h)$-convex functions can be seen in [10-21]. In [22], Pachpatte investigated the product of functions for developing Hermite-Hadamard-type inequalities by using the usual convexity. Recently, Noor et al. [23] introduced the idea of $(\alpha, m, h)$-convex function and established few fundamental inequalities for the class of twice-differentiable functions. Since the $(\alpha, m, h)$-convexity generalizes the concept of classical convexity, m-convexity, and $(\alpha, m)$-convex functions, the results therein are generalized. Also, the convex functions and their described generalizations are characterized by the product of functions in an elegant way. Motivated by these generalizations and $[1,3,22,23]$, we utilize the idea of product of functions in the setting of $(\alpha, m, h)$-convex function to establish Hermite-Hadamard-type inequalities for functions. We study different cases of double and triple integrals to derive some new results. This is the novel and innovative approach to 
characterize the convex function with the product of $h$-convex, $m$-convex, $(s, m)$-convex, and $(\alpha, s)$-convex functions. Our results unify several classes of functions like $(s, m)$-Godunova-Levin, $m$-Godunova-Levin, and $(\alpha, s)$-Godunova-Levin functions with mild conditions in an elegant way.

\section{Preliminaries}

This section is devoted to few well-known definitions fromthe literature. In [1], Toader gave the definition of $m$-convex function in the following manner.

Definition 1. A function $p: I \longrightarrow(0, \infty)$ is $m$-convex, if

$$
p\left(\eta x_{1}+m(1-\eta) x_{2}\right) \leq t p\left(x_{1}\right)+m(1-\eta) p\left(x_{2}\right),
$$

for all $x_{1}, x_{2} \in I, m \in[0,1]$, and $\eta \in[0,1]$.

In [24], Mihesan extended the idea of $m$-convex functions by introducing the idea of $(\alpha, m)$-convex function as follows.

Definition 2. A function $p: I \longrightarrow(0, \infty)$ is $(\alpha, m)$-convex, if

$$
p\left(\eta x_{1}+m(1-\eta) x_{2}\right) \leq \eta^{\alpha} p\left(x_{1}\right)+m\left(1-\eta^{\alpha}\right) p\left(x_{2}\right),
$$

for all $x_{1}, x_{2} \in I, \eta \in[0,1]$, and $(\alpha, m) \in[0,1]^{2}$.

In [23], Noor et al. generalized the idea of $m$-convexity and $(\alpha, m)$-convexity in a more general way with the definition of $(\alpha, m, h)$-convexity.

Definition 3. A function $p: I \longrightarrow(0, \infty)$ is $(\alpha, m, h)$-convex, if

$$
p\left(\eta x_{1}+m(1-\eta) x_{2}\right) \leq h\left(\eta^{\alpha}\right) p\left(x_{1}\right)+m h\left(1-\eta^{\alpha}\right) p\left(x_{2}\right),
$$

for all $x_{1}, x_{2} \in I, \eta \in[0,1],(\alpha, m) \in[0,1]^{2} \quad$ and $h:[0,1] \longrightarrow[0,1]$.

In [22], Pachpatte used the idea of product of functions for convex functions to establish the following result.
Theorem 1. Let $p$ and $q$ be nonnegative and convex functions on $\left[\xi_{1}, \xi_{2}\right]$ and further assume that they are real valued. Then, the following two inequalities hold:

$$
\begin{aligned}
\frac{1}{\xi_{2}-\xi_{1}} \int_{\xi_{1}}^{\xi_{2}} p(x) q(x) \mathrm{d} x \leq & \frac{1}{3} M\left(\xi_{1}, \xi_{2}\right)+\frac{1}{6} N\left(\xi_{1}, \xi_{2}\right), \\
2 p\left(\frac{\xi_{1}+\xi_{2}}{2}\right) q\left(\frac{\xi_{1}+\xi_{2}}{2}\right) \leq & \frac{1}{\xi_{2}-\xi_{1}} \int_{\xi_{1}}^{\xi_{2}} p(x) q(x) \mathrm{d} x \\
& +\frac{1}{6} M\left(\xi_{1}, \xi_{2}\right)+\frac{1}{3} N\left(\xi_{1}, \xi_{2}\right) .
\end{aligned}
$$

\section{Main Results}

This section contains the main results of our work involving product of $(\alpha, m, h)$-convex functions to obtain Hermite-Hadamard-type integral inequalities for two functions $p$ and $q$. These inequalities have also been studied for double and triple integrals.

Theorem 2. Assume that $p$ and $q$ are nonnegative and realvalued functions with $p q \in L\left[\xi_{1}, \xi_{2}\right]$, where $\xi_{1}, b \in I$ and $\xi_{1}<\xi_{2}$. Furthermore, assume that $p$ and $q$ are $(\alpha, m, h)$-convex on $\left[\xi_{1}, \xi_{2}\right]$. Then, we have the following inequality:

$\frac{1}{m \xi_{2}-\xi_{1}} \int_{\xi_{1}}^{m \xi_{2}} p(x) q(x) \mathrm{d} x \leq h\left(\xi_{1}, \xi_{1}\right) L+h\left(\xi_{2}, \xi_{2}\right) M+h\left(\xi_{1}, \xi_{2}\right) N$

Here,

$$
\begin{aligned}
& H\left(\xi_{1}, \xi_{1}\right)=p\left(\xi_{1}\right) q\left(\xi_{1}\right) \\
& H\left(\xi_{2}, \xi_{2}\right)=p\left(\xi_{2}\right) q\left(\xi_{2}\right) \\
& H\left(\xi_{1}, \xi_{2}\right)=p\left(\xi_{1}\right) q\left(\xi_{2}\right)+p\left(\xi_{2}\right) q\left(\xi_{1}\right) .
\end{aligned}
$$

Also, $L=\int_{0}^{1}\left[h\left(\eta^{\alpha}\right)\right]^{2} d \eta, M=\int_{0}^{1} m^{2}\left[h\left(1-\eta^{\alpha}\right)\right]^{2} d \eta$ and $N=\int_{0}^{1} m h\left(\eta^{\alpha}\right) h\left(1-\eta^{\alpha}\right) d \eta$.

Proof. Assume that $p$ and $q$ are $(\alpha, m, h)$-convex on $\left[\xi_{1}, \xi_{2}\right]$; then,

$$
\begin{aligned}
p\left(\eta \xi_{1}+m(1-\eta) \xi_{2}\right) q\left(\eta \xi_{1}+m(1-\eta) \xi_{2}\right) \leq & {\left[h\left(\eta^{\alpha}\right) p\left(\xi_{1}\right)+m h\left(1-\eta^{\alpha}\right) p\left(\xi_{2}\right)\right]\left[h\left(\eta^{\alpha}\right) q\left(\xi_{1}\right)+m h\left(1-\eta^{\alpha}\right) q\left(\xi_{2}\right)\right] } \\
= & {\left[h\left(\eta^{\alpha}\right)\right]^{2} p\left(\xi_{1}\right) q\left(\xi_{1}\right)+m^{2}\left[h\left(1-\eta^{\alpha}\right)\right]^{2} p\left(\xi_{2}\right) q\left(\xi_{2}\right)+m h\left(\eta^{\alpha}\right) \times } \\
& h\left(1-\eta^{\alpha}\right)\left[p\left(\xi_{1}\right) q\left(\xi_{2}\right)+p\left(\xi_{2}\right) q\left(\xi_{1}\right)\right] .
\end{aligned}
$$

Now,

$$
\begin{aligned}
& \int_{0}^{1} p\left(\eta \xi_{1}+m(1-\eta) \xi_{2}\right) q\left(\eta \xi_{1}+m(1-\eta) \xi_{2}\right) \mathrm{d} \eta \\
& \quad \leq\left[\begin{array}{c}
\int_{0}^{1}\left[h\left(\eta^{\alpha}\right)\right]^{2} p\left(\xi_{1}\right) q\left(\xi_{1}\right) d \eta+\int_{0}^{1} m^{2}\left[h\left(1-\eta^{\alpha}\right)\right]^{2} p\left(\xi_{2}\right) q\left(\xi_{2}\right) \mathrm{d} \eta \\
+\int_{0}^{1} m h\left(\eta^{\alpha}\right) h\left(1-\eta^{\alpha}\right)\left[p\left(\xi_{1}\right) q\left(\xi_{2}\right)+p\left(\xi_{2}\right) q\left(\xi_{1}\right)\right] \mathrm{d} \eta
\end{array}\right] .
\end{aligned}
$$


Thus,

$$
\begin{aligned}
& \int_{0}^{1} p\left(\eta \xi_{1}+m(1-\eta) \xi_{2}\right) q\left(\eta \xi_{1}+m(1-\eta) \xi_{2}\right) \mathrm{d} \eta \\
& \quad \leq\left[\begin{array}{c}
h\left(\xi_{1}, \xi_{1}\right) L+h\left(\xi_{2}, \xi_{2}\right) M \\
+h\left(\xi_{1}, \xi_{2}\right) N
\end{array}\right] .
\end{aligned}
$$

$$
\begin{aligned}
\frac{1}{m \xi_{2}-\xi_{1}} \int_{\xi_{1}}^{m \xi_{2}} p(x) q(x) \mathrm{d} x \leq & H\left(\xi_{1}, \xi_{1}\right) L+H\left(\xi_{2}, \xi_{2}\right) M \\
& +H\left(\xi_{1}, \xi_{2}\right) N .
\end{aligned}
$$

Now, substituting $\eta \xi_{1}+m(1-\eta) \xi_{2}=x$, we obtain

Corollary 1. If $h(\eta)=\eta$, then

$$
\frac{1}{m \xi_{2}-\xi_{1}} \int_{\xi_{1}}^{m \xi_{2}} p(x) q(x) \mathrm{d} x \leq\left[\begin{array}{c}
\frac{1}{2 \alpha+1} H\left(\xi_{1}, \xi_{1}\right)+\frac{2 m^{2} \alpha^{2}}{(2 \alpha+1)(\alpha+1)} H\left(\xi_{2}, \xi_{2}\right) \\
+\frac{m \alpha}{(2 \alpha+1)(\alpha+1)} H\left(\xi_{1}, \xi_{2}\right)
\end{array}\right]
$$

Remark 1. Corollary 1, along with $m=1$ and $\alpha=1$, gives inequality (5).
Theorem 3. For any two $(\alpha, m, h)$-convex functions $p$ and $q$ with $p q \in L\left[\xi_{1}, \xi_{2}\right]$, we have the following estimates:

$$
2 p\left(\frac{\xi_{1}+m \xi_{2}}{2}\right) q\left(\frac{\xi_{1}+m \xi_{2}}{2}\right) \leq\left[\begin{array}{l}
\int_{0}^{1} p\left(\eta \xi_{1}+n(1-\eta) \xi_{2}\right) q\left(\eta \xi_{1}+m(1-\eta) \xi_{2}\right) \mathrm{d} \eta \\
+\left[H\left(\xi_{1}, \xi_{1}\right)+n^{2} H\left(\xi_{2}, \xi_{2}\right)\right] S+\frac{1}{2}\left[H\left(\xi_{1}, \xi_{2}\right)\right] T
\end{array}\right]
$$

where

$$
\begin{aligned}
& S=\int_{0}^{1} h\left(\eta^{\alpha}\right) h\left(1-\eta^{\alpha}\right) \mathrm{d} \eta \\
& T=\int_{0}^{1} n\left[\left[h\left(\eta^{\alpha}\right)\right]^{2}+\left[h\left(1-\eta^{\alpha}\right)\right]^{2}\right] \mathrm{d} \eta .
\end{aligned}
$$

Also $H\left(\xi_{1}, \xi_{1}\right), H\left(\xi_{2}, \xi_{2}\right)$, and $H\left(\xi_{1}, \xi_{2}\right)$ are as defined in the above theorem.

$$
\begin{aligned}
p\left(\frac{\xi_{1}+m \xi_{2}}{2}\right) q\left(\frac{\xi_{1}+m \xi_{2}}{2}\right) & =\left[\begin{array}{c}
p\left(\frac{\eta \xi_{1}+m(1-\eta) \xi_{2}}{2}+\frac{(1-\eta) \xi_{1}+m \eta \xi_{2}}{2}\right) \times \\
q\left(\frac{\eta \xi_{1}+m(1-\eta) \xi_{2}}{2}+\frac{(1-\eta) \xi_{1}+m \eta \xi_{2}}{2}\right)
\end{array}\right] \\
\leq & {\left[\begin{array}{c}
\frac{1}{4}\left[p\left(\eta \xi_{1}+m(1-\eta) \xi_{2}\right)+p\left((1-\eta) \xi_{1}+m \eta \xi_{2}\right)\right] \times \\
{\left[q\left(\eta \xi_{1}+m(1-\eta) \xi_{2}+q\left((1-\eta) \xi_{1}+m \eta \xi_{2}\right)\right)\right]}
\end{array}\right] }
\end{aligned}
$$




$$
\begin{aligned}
& =\left[\begin{array}{c}
\frac{1}{4}\left[p\left(\eta \xi_{1}+m(1-\eta) \xi_{2}\right) q\left(\eta \xi_{1}+m(1-\eta) \xi_{2}\right)\right. \\
\left.+p\left((1-\eta) \xi_{1}+m \eta \xi_{2}\right) q\left((1-\eta) \xi_{1}+m \eta \xi_{2}\right)\right] \\
+\frac{1}{4}\left[p\left(\eta \xi_{1}+m(1-\eta) \xi_{2}\right) q\left((1-\eta) \xi_{1}+m \eta \xi_{2}\right)\right. \\
\left.+p\left((1-\eta) \xi_{1}+m \eta \xi_{2}\right) q\left(\eta \xi_{1}+m(1-\eta) \xi_{2}\right)\right]
\end{array}\right] \\
& \leq\left[\begin{array}{c}
\frac{1}{4}\left[p\left(\eta \xi_{1}+m(1-\eta) \xi_{2}\right) q\left(\eta \xi_{1}+m(1-\eta) \xi_{2}\right)\right] \\
\left.+p\left((1-\eta) \xi_{1}+m \eta \xi_{2}\right) q\left((1-\eta) \xi_{1}+m \eta \xi_{2}\right)\right] \\
+\frac{1}{4}\left[h\left(\eta^{\alpha}\right) p\left(\xi_{1}\right)+m h\left(1-\eta^{\alpha}\right) p\left(\xi_{2}\right)\right] \times \\
\left.+\frac{1}{4} m\left[h\left(\eta^{\alpha}\right)\right]^{2}+\left[h\left(1-\eta^{\alpha}\right)\right]^{2}\right]\left[p\left(\xi_{1}\right) q\left(\xi_{2}\right)+p\left(\xi_{2}\right) q\left(\xi_{1}\right)\right]
\end{array}\right] \\
& \leq\left[\begin{array}{c}
\frac{1}{4}\left[p\left(\eta \xi_{1}+m(1-\eta) \xi_{2}\right) q\left(\eta \xi_{1}+m(1-\eta) \xi_{2}\right)+p\left((1-\eta) \xi_{1}\right.\right. \\
+\frac{1}{2}\left[h\left(\eta^{\alpha}\right) h\left(1-\eta^{\alpha}\right)\right]\left[p\left(\xi_{1}\right) p\left(\xi_{2}\right)+m^{2} p\left(\xi_{2}\right) q\left(\xi_{2}\right)\right] \\
\left.\left.+m \eta \xi_{2}\right) q\left((1-\eta) \xi_{1}+m \eta \xi_{2}\right)\right]
\end{array}\right] \\
& \leq
\end{aligned}
$$

On integrating,

$$
p\left(\frac{\xi_{1}+m \xi_{2}}{2}\right) q\left(\frac{\xi_{1}+m \xi_{2}}{2}\right) \leq\left[\begin{array}{c}
\frac{1}{4} \int_{0}^{1}\left[p\left(\eta \xi_{1}+m(1-\eta) \xi_{2}\right) q\left(\eta \xi_{1}+m(1-\eta) \xi_{2}\right)\right. \\
\left.+p\left((1-\eta) \xi_{1}+m \eta \xi_{2}\right) q\left((1-\eta) \xi_{1}+m \eta \xi_{2}\right)\right] \mathrm{d} \eta \\
+\frac{1}{2} \int_{0}^{1} h\left(\eta^{\alpha}\right) h\left(1-\eta^{\alpha}\right) \mathrm{d} \eta\left[p\left(\xi_{1}\right) p\left(\xi_{2}\right)+m^{2} p\left(\xi_{2}\right) q\left(\xi_{2}\right)\right] \\
+\frac{1}{4} \int_{0}^{1}\left[m\left[h\left(\eta^{\alpha}\right)\right]^{2}\right]+\left[h\left(1-\eta^{\alpha}\right)\right]^{2} \mathrm{~d} \eta\left[p\left(\xi_{1}\right) q\left(\xi_{2}\right)+p\left(\xi_{2}\right) q\left(\xi_{1}\right)\right]
\end{array}\right] .
$$

Thus, we obtain

$$
p\left(\frac{\xi_{1}+m \xi_{2}}{2}\right) q\left(\frac{\xi_{1}+m \xi_{2}}{2}\right) \leq\left[\begin{array}{c}
\frac{1}{2} \int_{0}^{1} p\left(\eta \xi_{1}+m(1-\eta) \xi_{2}\right) q\left(\eta \xi_{1}+m(1-\eta) \xi_{2}\right) \mathrm{d} \eta \\
+\frac{1}{2}\left[H\left(\xi_{1}, \xi_{1}\right)+m H\left(\xi_{2}, \xi_{2}\right)\right] S+\frac{1}{4}\left[H\left(\xi_{1}, \xi_{2}\right)\right] T
\end{array}\right]
$$


Now, substituting $x=\eta \xi_{1}+n(1-\eta) \xi_{2}$, we obtain

$$
2 p\left(\frac{\xi_{1}+m \xi_{2}}{2}\right) q\left(\frac{\xi_{1}+m \xi_{2}}{2}\right) \leq\left[\frac{1}{\xi_{2}-\xi_{1}} \int_{\xi_{1}}^{\xi_{2}} p(x) q(x) \mathrm{d} \eta+\left[h\left(\xi_{1}, \xi_{1}\right)+n h\left(\xi_{2}, \xi_{2}\right)\right] S+\frac{1}{2}\left[h\left(\xi_{1}, \xi_{2}\right)\right] T\right] \text {. }
$$

Corollary 2. If $h(\eta)=\eta$, then

$$
2 p\left(\frac{\xi_{1}+m \xi_{2}}{2}\right) q\left(\frac{\xi_{1}+m \xi_{2}}{2}\right) \leq\left[\begin{array}{c}
\frac{1}{\xi_{2}-\xi_{1}} \int_{\xi_{1}}^{\xi_{2}} p(x) q(x) \mathrm{d} \eta+\frac{\alpha}{(\alpha+1)(2 \alpha+1)}\left[H\left(\xi_{1}, \xi_{1}\right)+m H\left(\xi_{2}, \xi_{2}\right)\right]+\frac{m\left(\alpha^{2}+\alpha+1\right)}{2(\alpha+1)(2 \alpha+1)} \\
+\frac{m\left(\alpha^{2}+\alpha+1\right)}{2(\alpha+1)(2 \alpha+1)} H\left(\xi_{1}, \xi_{2}\right)
\end{array}\right]
$$

Remark 2. Corollary 2, along with $m=1$ and $\alpha=1$, gives inequality (6).
Theorem 4. Assume that $p$ and $q$ are $(\alpha, m, h)$-convex functions satisfying all the conditions of the above theorem; then, the following inequality holds:

$$
\begin{aligned}
& \frac{1}{\left(\xi_{2}-\xi_{1}\right)^{2}} \int_{\xi_{1}}^{\xi_{2}} \int_{\xi_{1}}^{\xi_{2}} \int_{0}^{1} p(\eta x+m(1-\eta) y) q(\eta x+m(1-\eta) y) \mathrm{d} \eta \mathrm{d} y \mathrm{~d} x \\
& \quad \leq[L+M] \frac{1}{\xi_{2}-\xi_{1}} \int_{\xi_{1}}^{\xi_{2}} p(x) q(x) \mathrm{d} x+\frac{1}{2} \frac{1}{\left(\xi_{2}-\xi_{1}\right)^{2}} N\left[H\left(\xi_{1}, \xi_{1}\right)+H\left(\xi_{2}, \xi_{2}\right)+H\left(\xi_{1}, \xi_{2}\right)\right],
\end{aligned}
$$

where

Proof. Using the $(\alpha, m, h)$-convexity,

$$
\begin{aligned}
L & =\int_{0}^{1}\left[h\left(\eta^{\alpha}\right)\right]^{2} \mathrm{~d} \eta \\
M & =\int_{0}^{1} m^{2}\left[h\left(1-\eta^{\alpha}\right)\right]^{2} \mathrm{~d} \eta, \\
N & =\int_{0}^{1} m h\left(\eta^{\alpha}\right) h\left(1-\eta^{\alpha}\right) \mathrm{d} \eta .
\end{aligned}
$$

$$
p(\eta x+m(1-\eta) y) q(\eta x+m(1-\eta) y) \leq\left[\begin{array}{c}
{\left[h\left(\eta^{\alpha}\right) p(x)+m h\left(1-\eta^{\alpha}\right) p(y)\right] \times} \\
{\left[h\left(\eta^{\alpha}\right) q(x)+m h\left(1-\eta^{\alpha}\right) q(y)\right]} \\
=\left[h\left(\eta^{\alpha}\right)\right]^{2} p(x) q(x)+m^{2}\left[h\left(1-\eta^{\alpha}\right)\right] \times 2 p(y) q(y) \\
+\left[m h\left(\eta^{\alpha}\right) h\left(1-\eta^{\alpha}\right)\right][p(x) q(y)+p(y) q(x)]
\end{array}\right]
$$

Integrating on $[0,1]$, we obtain

$$
\begin{aligned}
\int_{0}^{1} p(\eta x+n(1-\eta) y) q(\eta x+m(1-\eta) y) d \eta \leq & p(x) q(x) \int_{0}^{1}\left[h\left(\eta^{\alpha}\right)\right]^{2} \mathrm{~d} \eta+p(y) q(y) \int_{0}^{1} m^{2}\left[h\left(1-\eta^{\alpha}\right)\right]^{2} \mathrm{~d} \eta \\
& +[p(x) q(y)+p(y) q(x)] \int_{0}^{1} m h\left(\eta^{\alpha}\right) h\left(1-\eta^{\alpha}\right) \mathrm{d} \eta
\end{aligned}
$$


Thus,

Now, integrating on the rectangle $[0,1] \times[0,1]$,

$\int_{0}^{1} p(\eta x+m(1-\eta) y) q(\eta x+m(1-\eta) y) \mathrm{d} \eta$

$\leq[p(x) q(x)] L+[p(y) q(y)] M+[p(x) q(y)+p(y) q(x)] N$.

$$
\begin{aligned}
& \int_{\xi_{1}}^{\xi_{2}} \int_{\xi_{1}}^{\xi_{2}} \int_{0}^{1} p(\eta x+m(1-\eta) y) q(\eta x+m(1-\eta) y) \mathrm{d} \eta \mathrm{d} y \mathrm{~d} x \\
& \quad \leq L\left(\xi_{2}-\xi_{1}\right) \int_{\xi_{1}}^{\xi_{2}} p(x) q(x) \mathrm{d} x+M\left(\xi_{2}-\xi_{1}\right) \int_{\xi_{1}}^{\xi_{2}} p(y) q(y) \mathrm{d} y+N\left[\int_{\xi_{1}}^{x i_{2}} p(x) \mathrm{d} x \times \int_{\xi_{1}}^{\xi_{2}} q(y) \mathrm{d} y\right. \\
& \left.\quad+\int_{\xi_{1}}^{\xi_{2}} p(y) \mathrm{d} y \times \int_{\xi_{1}}^{\xi_{2}} q(x) \mathrm{d} x\right] .
\end{aligned}
$$

Now, applying Hadamard's inequality from right half to the above equation,

$$
\begin{aligned}
& \int_{\xi_{1}}^{\xi_{2}} \int_{\xi_{1}}^{\xi_{2}} \int_{0}^{1} p(\eta x+m(1-\eta) y) q(\eta x+m(1-\eta) y) \mathrm{d} \eta \mathrm{d} y \mathrm{~d} x \\
& \quad \leq[L+M]\left(\xi_{2}-\xi_{1}\right) \int_{\xi_{1}}^{\xi_{2}} p(x) q(x) \mathrm{d} x+\frac{1}{2} N\left[p\left(\xi_{1}\right) q\left(\xi_{1}\right)+p\left(\xi_{2}\right) q\left(\xi_{2}\right)+p\left(\xi_{1}\right) q\left(\xi_{2}\right)+p\left(\xi_{2}\right) q\left(\xi_{1}\right)\right] \\
& \quad=[L+M]\left(\xi_{2}-\xi_{1}\right) \int_{\xi_{1}}^{\xi_{2}} p(x) q(x) \mathrm{d} x+\frac{1}{2} N\left[H\left(\xi_{1}, \xi_{1}\right)+H\left(\xi_{2}, \xi_{2}\right)+H\left(\xi_{1}, \xi_{2}\right)\right] .
\end{aligned}
$$

Thus, we obtain

$$
\begin{aligned}
& \frac{1}{\left(\xi_{2}-\xi_{1}\right)^{2}} \int_{\xi_{1}}^{\xi_{2}} \int_{\xi_{1}}^{\xi_{2}} \int_{0}^{1} p(\eta x+m(1-\eta) y) q(\eta x+m(1-\eta) y) \mathrm{d} \eta \mathrm{d} y \mathrm{~d} x \\
& \quad \leq \frac{1}{\xi_{2}-\xi_{1}}[L+M] \int_{\xi_{1}}^{\xi_{2}} p(x) q(x) \mathrm{d} x+\frac{1}{2} \frac{1}{\left(\xi_{2}-\xi_{1}\right)^{2}} N\left[H\left(\xi_{1}, \xi_{1}\right)+H\left(\xi_{2}, \xi_{2}\right)+H\left(\xi_{1}, \xi_{2}\right)\right] .
\end{aligned}
$$

Corollary 3. For $h(\eta)=\eta$, we have

$$
\begin{aligned}
& \frac{\left.1 \xi_{2}-\xi_{1}\right)}{2} \int_{\xi_{1}}^{\xi_{2}} \int_{\xi_{1}}^{\xi_{2}} \int_{0}^{1} p(\eta x+m(1-\eta y) q(\eta x+n(1-\eta y) \mathrm{d} \eta \mathrm{d} y \mathrm{~d} x \\
& \leq\left[\begin{array}{c}
\frac{2 \alpha^{2} m^{2}+\alpha+1}{(2 \alpha+1)(\alpha+1)\left(\xi_{2}-\xi_{1}\right)} \int_{\xi_{1}}^{\xi_{2}} p(x) q(x) \mathrm{d} x \\
+\frac{m \alpha}{2(2 \alpha+1)(\alpha+1)\left(\xi_{2}-\xi_{1}\right)^{2}}\left[H\left(\xi_{1}, \xi_{1}\right)+H\left(\xi_{2}, \xi_{2}\right)+H\left(\xi_{1}, \xi_{2}\right)\right]
\end{array}\right] .
\end{aligned}
$$


Theorem 5. Assume that $p$ and $q$ are nonnegative realvalued functions such that they are $(\alpha, m, h)$-convex and $p q \in L\left[\xi_{1}, \xi_{2}\right]$; then,

$$
\begin{aligned}
& \frac{1}{\xi_{2}-\xi_{1}} \int_{\xi_{1}}^{\xi_{2}} \int_{0}^{1} p\left(\eta x+m(1-\eta) \frac{\xi_{1}+\xi_{2}}{2}\right) q\left(\eta x+m(1-\eta) \frac{\xi_{1}+\xi_{2}}{2}\right) \mathrm{d} \eta \mathrm{d} x \\
& \quad \leq \frac{L}{\xi_{2}-\xi_{1}} \int_{\xi_{1}}^{\xi_{2}} p(x) q(x) \mathrm{d} x+\frac{1}{4} M\left[H\left(\xi_{1}, \xi_{1}\right)+H\left(\xi_{2}, \xi_{2}\right)+H\left(\xi_{1}, \xi_{2}\right)\right]+\frac{N}{2\left(\xi_{2}-\xi_{1}\right)}\left[H\left(\xi_{1}, \xi_{1}\right)+H\left(\xi_{2}, \xi_{2}\right)+H\left(\xi_{1}, \xi_{2}\right)\right]
\end{aligned}
$$

where $L, M$, and $N$ are as in the above theorem.

Proof. Since $p$ and $q$ are $(\alpha, m, h)$-convex,

$$
\begin{aligned}
& p\left(\eta x+m(1-\eta)\left(\frac{\xi_{1}+\xi_{2}}{2}\right)\right) q\left(\eta x+m(1-\eta)\left(\frac{\xi_{1}+\xi_{2}}{2}\right)\right) \\
& \leq {\left[h\left(\eta^{\alpha}\right) p(x)+m h\left(1-\eta^{\alpha}\right) p\left(\frac{\xi_{1}+\xi_{2}}{2}\right)\right]\left[h\left(\eta^{\alpha}\right) q(x)+m h\left(1-\eta^{\alpha}\right) q\left(\frac{\xi_{1}+\xi_{2}}{2}\right)\right] } \\
&= {\left[\left[h\left(\eta^{\alpha}\right)\right]^{2} p(x) q(x)+m^{2}\left[h\left(1-\eta^{\alpha}\right)\right]^{2} p\left(\frac{\xi_{1}+\xi_{2}}{2}\right) q\left(\frac{\xi_{1}+\xi_{2}}{2}\right)\right] } \\
&+m h\left(\eta^{\alpha}\right) h\left(1-\eta^{\alpha}\right)\left[p(x) q\left(\frac{\xi_{1}+\xi_{2}}{2}\right)+q(x) p\left(\frac{\xi_{1}+\xi_{2}}{2}\right)\right] .
\end{aligned}
$$

This implies that

$$
\begin{aligned}
\int_{0}^{1} p\left(\eta x+n(1-\eta)\left(\frac{\xi_{1}+\xi_{2}}{2}\right)\right) q\left(\eta x+m(1-\eta)\left(\frac{\xi_{1}+\xi_{2}}{2}\right)\right) \mathrm{d} \eta \\
\leq p(x) q(x) \int_{0}^{1}\left[h\left(\eta^{\alpha}\right)\right]^{2} \mathrm{~d} \eta+p\left(\frac{\xi_{1}+\xi_{2}}{2}\right) q\left(\frac{\xi_{1}+\xi_{2}}{2}\right) \int_{0}^{1} m^{2}\left[h\left(1-\eta^{\alpha}\right)\right]^{2} \mathrm{~d} \eta \\
\quad+\left[p(x) q\left(\frac{\xi_{1}+\xi_{2}}{2}\right)+q(x) p\left(\frac{\xi_{1}+\xi_{2}}{2}\right)\right] \int_{0}^{1} m h\left(\eta^{\alpha}\right) h\left(1-\eta^{\alpha}\right) \mathrm{d} \eta .
\end{aligned}
$$

Integrating on $[0,1]$,

$$
\begin{aligned}
& \int_{\xi_{1}}^{\xi_{2}} \int_{0}^{1} p\left(\eta x+n(1-\eta)\left(\frac{\xi_{1}+\xi_{2}}{2}\right)\right) q\left(\eta x+m(1-\eta)\left(\frac{\xi_{1}+\xi_{2}}{2}\right)\right) \mathrm{d} \eta \mathrm{d} x \\
& \quad \leq L \int_{\xi_{1}}^{\xi_{2}} p(x) q(x) \mathrm{d} x+M\left(\xi_{2}-\xi_{1}\right) p\left(\frac{\xi_{1}+\xi_{2}}{2}\right) q\left(\frac{\xi_{1}+\xi_{2}}{2}\right)+\left[q\left(\frac{\xi_{1}+\xi_{2}}{2}\right) \int_{\xi_{1}}^{\xi_{2}} p(x) \mathrm{d} x\right. \\
& \left.\quad+p\left(\frac{\xi_{1}+\xi_{2}}{2}\right) \int_{\xi_{1}}^{\xi_{2}} q(x) \mathrm{d} x\right] N .
\end{aligned}
$$


Now, using the right half of Hadamard's inequality on the above equation,

$$
\begin{aligned}
& \int_{\xi_{1}}^{\xi_{2}} \int_{0}^{1} p\left(\eta x+n(1-\eta)\left(\frac{\xi_{1}+\xi_{2}}{2}\right)\right) q\left(\eta x+m(1-\eta)\left(\frac{\xi_{1}+\xi_{2}}{2}\right)\right) \mathrm{d} v \mathrm{~d} x \\
& \leq L \int_{\xi_{1}}^{\xi_{2}} p(x) q(x) \mathrm{d} x+M\left(\xi_{2}-\xi_{1}\right)\left[\frac{p\left(\xi_{1}\right)+p\left(\xi_{2}\right)}{2} \frac{q\left(\xi_{1}\right)+q\left(\xi_{2}\right)}{2}\right] \\
& \quad+\left[2 \frac{p\left(\xi_{1}\right)+p\left(\xi_{2}\right)}{2} \frac{q\left(\xi_{1}\right)+q\left(\xi_{2}\right)}{2}\right] N .
\end{aligned}
$$

Hence, we obtain

$$
\begin{aligned}
& \frac{1}{\xi_{2}-\xi_{1}} \int_{\xi_{1}}^{\xi_{2}} \int_{0}^{1} p\left(\eta x+m(1-\eta)\left(\frac{\xi_{1}+\xi_{2}}{2}\right)\right) q\left(\eta x+n(1-\eta)\left(\frac{\xi_{1}+\xi_{2}}{2}\right)\right) \mathrm{d} \eta \mathrm{d} x \\
& \leq \frac{L}{\xi_{2}-\xi_{1}} \int_{\xi_{1}}^{\xi_{2}} p(x) q(x) \mathrm{d} x+\frac{M}{4}\left[H\left(\xi_{1}, \xi_{1}\right)+H\left(\xi_{2}, \xi_{2}\right)+H\left(\xi_{1}, \xi_{2}\right)\right] \\
& \quad+\frac{N}{2\left(\xi_{2}-\xi_{1}\right)}\left[h\left(\xi_{1}, \xi_{1}\right)+h\left(\xi_{2}, \xi_{2}\right)+h\left(\xi_{1}, \xi_{2}\right)\right] .
\end{aligned}
$$

This completes the proof.

Corollary 4. If $h(\eta)=\eta$, then

$$
\begin{aligned}
& \frac{1}{\xi_{2}-\xi_{1}} \int_{\xi_{1}}^{\xi_{2}} \int_{0}^{1} p\left(\eta x+n(1-\eta)\left(\frac{\xi_{1}+\xi_{2}}{2}\right)\right) q\left(\eta x+n(1-\eta)\left(\frac{\xi_{1}+\xi_{2}}{2}\right)\right) \mathrm{d} \eta \mathrm{d} x \\
& \leq \frac{1}{\left(\xi_{2}-\xi_{1}\right)(2 \alpha+1)} \int_{\xi_{1}}^{\xi_{2}} p(x) q(x) \mathrm{d} x+\frac{\alpha^{2} m^{2}}{2(\alpha+1)(2 \alpha+1)}\left[h\left(\xi_{1}, \xi_{1}\right)+h\left(\xi_{2}, \xi_{2}\right)+h\left(\xi_{1}, \xi_{2}\right)\right] \\
& \quad+\frac{m \alpha}{2\left(\xi_{2}-\xi_{1}\right)(\alpha+1)(2 \alpha+1)}\left[h\left(\xi_{1}, \xi_{1}\right)+h\left(\xi_{2}, \xi_{2}\right)+h\left(\xi_{1}, \xi_{2}\right)\right] .
\end{aligned}
$$

Remark 4. For $\alpha=1=m$, we obtain equation (4) of [22].

\section{Conclusion}

In this paper, we utilize the product of functions to develop the class of generalized convex functions using two given functions. We have studied this product for $(\alpha, m, h)$-convex functions. Afterwards, we applied this to investigate Hermite-Hadamard inequalities of various types. We have analyzed that for the specific value of $h$, that is, to be identity function, these results coincide with the results for the product of $(\alpha, m)$-convex functions. Moreover, the results are true for the said product in the sense of $\mathrm{m}$-convexity with $\alpha=1$. The comparison reflects that the obtained results improve and generalize the results for convex, $m$-convex, and $(\alpha, m)$-convex functions in a peculiar way. For further interest of the readers in this direction, one may examine this product for invex, preinvex, m-preinvex, harmonically preinvex, and logarithmically preinvex functions. The idea is also interesting for fractional integrals and stochastic process for convex functions for new aspects in this regard [25].

\section{Data Availability}

No data were used to support this study.

\section{Conflicts of Interest}

The authors declare that they have no conflicts of interest. 


\section{Acknowledgments}

This research was supported by the Ministry of Higher Education Malaysia, under FRGS Grant No: K179.

\section{References}

[1] G. Toader, "Some generalization of convexity approximation," in Proceedings of the Colloquium on Approximation and Optimization, Technical University of Cluj-Napoca, ClujNapoca, Romania, 1985.

[2] M. K. Bakula, M. E. Özdemir, and J. Pecaric, "Hadamard type inequalities for m-convex and $(\alpha, m)$-convex functions," Journal of Inequalities in Pure and Applied Mathematics, vol. 9, no. 4, 12 pages, 2008.

[3] S. S. Dragomir and G. Toader, "Some inequalities for $m$-convex functions," Studia Universitatis Babeş-Bolyai Mathematica, vol. 38, pp. 21-28, 1993.

[4] S. S. Dragomir, "On some new inequalities of HermiteHadamard type for $\$ \mathrm{~m} \$$-- convex functions," Tamkang Journal of Mathematics, vol. 33, no. 1, pp. 45-56, 2002.

[5] H.-P. Yin and F. Qi, "Hermite-Hadamard type inequalities for product of $(\alpha, m)$-convex functions," Journal of Nonlinear Science and Applications, vol. 8, pp. 231-236, 2015.

[6] I. Iscan, "New estimates on generalization of some integral inequalities for $(\alpha, m)$-convex functions," Contemporary Analysis and Applied Mathematics, vol. 1, no. 2, pp. 253-264, 2013.

[7] S. Varosanec, Journal of Mathematical Analysis and Applications, vol. 326, pp. 303-311, 2007.

[8] S.-H. Wang, B.-Y. Xi, and F. Qi, "Some new inequalities of Hermite-Hadamard type for n-time differentiable functions which arem-convex," Analysis, vol. 32, no. 3, pp. 247-262, 2012.

[9] S.-H. Wang, B.-Y. Xi, and F. Qi, "On hermite-hadamard type inequalities for ( $\alpha, \mathrm{M})$-convex functions," International Journal of Open Problems in Computer Science and Mathematics, vol. 5, no. 4, pp. 47-56, 2012.

[10] R. F. Bai, F. Qi, and B. Y. Xi, "Hermite-Hadamard type inequalities for the $m$-and $(\alpha, m)$-logarithmically convex functions," Filomat, vol. 27, no. 1, pp. 1-7, 2013.

[11] M. K. Bakula, J. Peccarić, and M. Ribićić, "Companion inequalities to Jensen's inequality for m-convex and $(\alpha, m)$-convex functions," Journal of Inequalities in Pure and Applied Mathematics, vol. 7, no. 5, 2006.

[12] A. M. Bruckner and E. Ostrow, "Some function classes related to class of convex functions," Pacific Journal of Mathematics, vol. 4, pp. 1203-1215, 1962.

[13] H. Budak and M. Z. Sarıkaya, "Some new generalized Hermite-Hadamard inequalities for generalized convex functions and applications," Journal of Mathematical Extension, vol. 12, no. 4, pp. 51-66, 2018.

[14] P. Cerone, S. S. Dragomir, and J. Roumeliotis, "Some Ostrowski type inequalities for n-time differentiable mappings and applications," RGMIA, vol. 1, no. 1, , 1998, http:// rgmia.org/v1n1.php.

[15] P. Cerone, S. S. Dragomir, and J. Roumelotis, "Some Ostrowski type inequalities for n-time differentiable mappings and applications," Demonstratio Mathematica, vol. 32, no. 4, pp. 697-712, 1999.

[16] S. S. Dragomir and S. Fitzpatrick, "The Hadamard's inequality for $s$-convex functions in the second sense," Demonstratio Mathematica, vol. 32, pp. 687-696, 1999.
[17] S. S. Dragomir and C. E. M. Pearce, "Selected topics on Hermite-Hadamard inequalities and applications," RGMIA Monographs. Victoria University, 2000.

[18] M. Kunt and İ. İşcan, "Hermite-Hadamard-Fejér type inequalities for p-convex functions," Arab Journal of Mathematical Sciences, vol. 23, no. 2, pp. 215-230, 2017.

[19] M. Kunt and I. Iscan, "Hermite-Hadamard type inequalities for harmonically $(\alpha, m)$-convex functions by using fractional integrals," Konuralp Journal of Mathematics, vol. 5, no. 1, pp. 201-213, 2017.

[20] M. A. Latif, "Inequalities of Hermite-Hadamard type for functions whose derivatives in absolute value are convex with applications," Arab Journal of Mathematical Sciences, vol. 21, no. 1, pp. 84-97, 2015.

[21] M. A. Latif, S. S. Dragomir, and E. Momoniat, "On Hermite-Hadamard type integral inequalities for n-times differentiable $m$-and $(\alpha, m)$-ogarithmically convex functions," Filomat, vol. 30, no. 11, pp. 3101-3114, 2016.

[22] B. G. Pachpatte, "On some inequalities for convex functions," RGMIA, vol. 6, 2003, http://rgmia.org/v6(E).php.

[23] M. A. Noor, M. U. Awan, K. I. Noor, and T. M. Rassias, "On $(\alpha, m, h)$-convexity," Applied Mathematics \& Information Sciences Letters, vol. 12, no. 1, pp. 145-150, 2018.

[24] V. G. Mihesan, "A generalization of the convexity," in Proceedings of the Seminar on Functional Equations Approximation, Cluj-Napoca, Romania, 1993.

[25] I. Iscan, "A new generalization of some integral inequalities for $(\alpha, m)$-convex functions," Mathematical Sciences, vol. 7 , no. 22, pp. 1-8, 2013. 